Oncogene (2004) 23, 4194-4195

(C) 2004 Nature Publishing Group All rights reserved 0950-9232/04 $\$ 30.00$

\title{
Guest Editor
}

Oncogene (2004) 23, 4194-4195. doi:10.1038/sj.onc.1207624

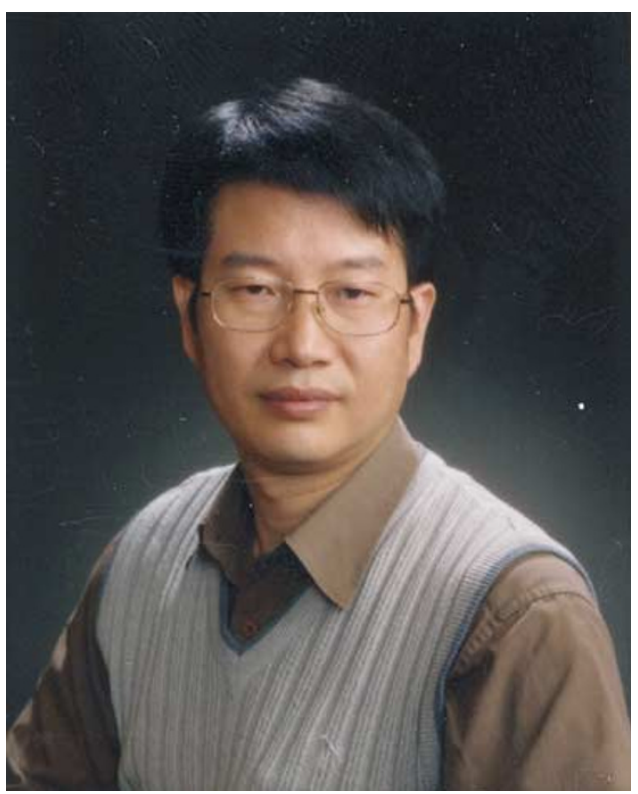

Dr Suk-Chul Bae
Suk-Chul Bae earned his Ph.D. degree in 1991 from Seoul National University College of Pharmacy. He is currently affiliated to the Chungbuh National University, South Korea. He is Director at the Tumor Suppressor Research Center of Ministry of Science and Technology of Korea since July 2003 and at the Institute for Tumor Research of Chungbuk National University since October 2002. He did his postdoctoral training at Ecole Normal Superiole, Lyon, France (October 1994-1995) and at the Kyoto University, Kyoto, Japan (October 1991-1994). He won the Korean Scientist of the Month award in 2002. He has many publications to his credit: Oncogenes as transcription Regulators: The Runt Transcription Factor, PEBP2/ CBF, and its involvement in Human Leukemia (1997) Birkhauser Verlag: Baser/Switzerland.

\section{Recent Publications}

Kim HJ, Kim JH, Bae SC, Choi SC, Kim HJ and Ryoo HM. (2002). The protein kinase $C$ pathway plays a central role in the FGF-stimulated expression and transactivation activity of Runx2. J. Biol. Chem., 278, 319-326.

Ichiro taniuchi, Motomi Osato, Takeshi Egawa, Mary Jean Sunshinel, Suk-Chul Bae, Toshihisa Komori, Yoshiaki Ito and Dan Littman R. (2002). Differential requirements for runx proteins in CD4 repression and epigenetic silencing during T lymphocyte development. Cell, 111, 621-633.

Inoue K, Ozaki S, Shiga T, Ito K, Masuda T, Okado N, Iseda T, Kawaguchi S, Ogawa M, Bae SC, Yamashita N, Itohara S, Kudo N and Ito Y. (2002). Runx3 controls the axonal projection of proprioceptive dorsal root ganglion neurons. Nat. Neurosci., 5 (10), 946-954.

Kyeong-Sook Lee, Seung-Hyun Hong and Suk-Chul Bae. (2002). Both the Smad and p38 MAPK pathways play a crucial role in Runx2 expression following induction by transforming growth factor-b and bone morphogenetic Protein. Oncogene, 21, 7156-7163.

Qing-Lin Li, Kosei Ito, Chohei Sakakura, Hiroshi Fukamachi, Ken-ichi Inoue, Xin-Zi Chi, Kwang-Youl Lee, Shintaro Nomura, Chang-Woo Lee, Sang-Bae Han, Hwan-Mook Kim, Wun-Jae Kim, Hiromitsu Yamamoto, Namiko Yamashita, Takashi Yano, Toshio Ikeda, Shigeyoshi Itohara, Johji Inazawa, Tatsuo Abe, Akeo Hagiwara, Hisakazu Yamagishi, Asako Ooe, Atsushi Kaneda, Takashi Sugimura,
Toshikazu Ushijima, Suk-Chul Bae and Yoshiaki Ito. (2002). Causal relationship between the loss of RUNX3 expression and gastric cancer. Cell, 109, 113-124.

Seung-Hee Nam, Yun-Hye Jin, Qing-Lin Li, Kwang-Youl Lee, Goo-Bo Jeong, Yoshiaki Ito, Junho Lee and Suk-Chul Bae. (2002). Expression pattern, regulation, and biological role of runt domain transcription factor, run, in Caenorhabditis elegans. Mol Cell. Biol., 22, 457-554.

Takahiko Sakuma, Qing-Lin Lib, Youngho Jin, Lak-Wan Choi, Eung-Gook Kim, Kosei Ito, Yoshiaki Ito, Shintaro Nomura and Suk-Chul Bae. (2001). Cloning and expression pattern of a novel PEBP2b-binding protein (charged amino acid rich leucine zipper [Crl-1]) in the mouse. Mech. of Dev., 104, 151-154.

Suk-Chul Bae, Kyung-Sook Lee, Yu-Wen Zhang and Yoshiaki Ito. (2001). Intimate relationship between TGF-b/BMP signaling and Runt domain transcription factor, PEBP2/ CBF. J. Bone and Joint Surg., 83-A, 48-55.

Kyeong-Sook Lee, Hyun-Jung Kim, Qing-Lin Li, Xin-Zi Chi, Chisato Ueta, Toshihisa Komori, John M Wozney, EungGook Kim, Je-Young Choi, Hyun-Mo Ryoo and Suk-Chul Bae. (2000). Runx2/PEBP2aA/Cbfa1 is a common target of TGF-b1 and BMP-2 and cooperation between Runx2 and Smad5 induces osteoblast specific gene expression in the pluripotent mesenchymal precursor cell line $\mathrm{C} 2 \mathrm{C} 12$. Mol. Cell. Biol., 20 (23), 8783-8792. 
Suk-Chul Bae and Junho Lee. (2000). cDNA cloning of run, a C. elegans Runt domain encoding gene. Gene, 241, 255-258. Hyeon Jin Park, Kyeong Eun Lee, Jung Min Um, Soo Young Choe, Xin Zi Chi, Jun Ah Lee, Hee Young Shin, Hyo Seop
Ahn and Suk-Chul Bae. (2000). Molecular detection of TELAML1 transcripts as a diagnostic tools and for monitoring of minimal residual disease in B-lineage childhood acute lymphoblastic leukemia. Mol. Cells, 10, 90-95. 